\title{
COMA IRREVERSÍVEL EM PACIENTE PORTADORA DE ESQUIZOFRENIA PARANOIDE: ENCEFALOPATIA DE WERNICKE APÓS SÍNDROME DE REALIMENTAÇÃO - UM RELATO DE CASO
}

\author{
IRREVERSIBLE COMA IN A PATIENT WITH PARANOID \\ SCHIZOPHRENIA: WERNICKE ENCEPHALOPATHY \\ AFTER REFEEDING SYNDROME - A CASE REPORT
}

Clin Biomed Res. 2020;40(4):242-246

1 Comissão de Suporte Nutricional, Hospital de Clínicas de Porto Alegre. Porto Alegre, RS, Brasil.

2 Hospital de Clínicas de Porto Alegre. Porto Alegre, RS, Brasil.

3 Serviço de Neurologia, Hospital de Clínicas de Porto Alegre. Porto Alegre, RS, Brasil.

Autor correspondente: Ana Carolina Peçanha Antonio aantonio@hcpa.edu.br Comissão de Suporte Nutricional, Hospital de Clínicas de Porto Alegre. Rua Ramiro Barcelos, 2350. 90035-903, Porto Alegre, RS, Brasil

\section{Ana Carolina Peçanha Antonio ${ }^{1}$, Maicon Cigolini², Thais Leite Secchi Juliana Mariano da Rocha Bandeira de Mello² \\ RESUMO}

Encefalopatia de Wernicke (EW) e síndrome de realimentação (SR) são duas condições frequentemente coexistentes, subdiagnosticadas e que podem implicar prognóstico reservado após sua instalação. Sua identificação precoce representa um desafio para os intensivistas, haja vista a falta de sensibilidade e especificidade das manifestações clínicas. Apresenta-se um relato de caso de uma paciente portadora de esquizofrenia paranoide, sem histórico de abuso de álcool, que desenvolveu quadro de coma irreversível após greve de fome, sendo feito diagnóstico tardio de EW associada a SR. Descreve-se a evolução clínica e neuropsiquiátrica com o intuito de enfatizar a necessidade crucial de alta suspeição diagnóstica, com reposição vitamínica imediata, vigilância de distúrbios eletrolíticos e progressão parcimoniosa do aporte nutricional.

Palavras-chave: Encefalopatia de Wernicke; síndrome da realimentação; inanição; transtornos psicóticos

\begin{abstract}
Wernicke encephalopathy (WE) and refeeding syndrome (RFS) are two often coexisting, underdiagnosed conditions that may involve a poor prognosis after their onset. Early identification represents a challenge for intensivists, given the lack of sensitivity and specificity of clinical manifestations. We report a case of a patient with paranoid schizophrenia, without a history of alcohol abuse, who developed irreversible coma after a hunger strike, with a late diagnosis of WE associated with RFS. The clinical and neuropsychiatric outcomes are described herein in order to emphasize the crucial need for a high diagnostic suspicion, with immediate vitamin replacement, monitoring of electrolyte disorders, and gradual progression of nutritional support.
\end{abstract}

Keywords: Wernicke encephalopathy, refeeding syndrome, starvation, psychotic disorders

\section{INTRODUÇÃO}

Inanição e desnutrição proteico-calórica são condições clínicas correlacionadas com o desenvolvimento de encefalopatia de Wernicke (EW) em pacientes não alcoolistas e frequentemente negligenciada em diagnósticos diferenciais de coma $^{1,2}$. A síndrome é desencadeada pela deficiência de tiamina (vitamina B1), sendo classicamente reconhecida pela tríade alteração do estado mental, oftalmoplegia e ataxia ${ }^{3}$. Além disso, indivíduos submetidos a privação de alimentos estão particularmente suscetíveis ao desenvolvimento de síndrome de realimentação (SR), que por si só exacerba a depleção da vitamina ${ }^{4}$. 


\section{RELATO DO CASO}

Paciente feminina, 48 anos, natural e procedente de Porto Alegre/RS, com história de esquizofrenia com cerca de 10 anos de diagnóstico, acompanhamento psiquiátrico regular, foi conduzida pelo pai a unidade de pronto-atendimento com queixa de surto psicótico. Havia cerca de 1 mês, a paciente iniciara com conduta isolacionista e recusa alimentar, mantendo discurso de que sua comida estava envenenada - delírio de contaminação. O pai referia algum grau de perda ponderal, embora não soubesse estimá-la. Negava tabagismo, etilismo ou outra forma de abuso de substância. Até então, apresentava vida funcional parcialmente independente, realizava tarefas domésticas básicas, e os medicamentos ficavam sob controle dos pais.

No pronto-atendimento, observou-se bradpsiquismo e rigidez muscular difusa e leve. Foi iniciada antibioticoterapia empiricamente frente à hipótese de infecção como fator precipitante da descompensação do quadro neuropsiquiátrico, e se solicitou transferência para unidade psiquiátrica. Com a piora progressiva do nível e conteúdo de consciência, após 6 dias de seguimento clínico, foi encaminhada à UTI onde se procedeu à intubação orotraqueal e instalação de ventilação mecânica invasiva e de sonda alimentar para nutrição enteral. Manteve-se aporte calórico recomendado para doente crítico: $25 \mathrm{kcal} / \mathrm{kg}$ de peso atual e $1,5 \mathrm{~g}$ de proteínas por $\mathrm{kg}$ de peso atual. Naquele momento, o exame neurológico apontava escala de coma de Glasgow igual a 3, miose pupilar bilateral sem fotorreação, com desconjugação do olhar vertical, hipotonia e arreflexia dos quatro membros e ausência de rigidez de nuca. Mantinha-se afebril e sem outros comemorativos hemodinâmicos. Parâmetros antropométricos registrados nesse momento foram de $59,3 \mathrm{kgs}$ e $1,66 \mathrm{~m}$ de altura, perfazendo índice de massa corporal (IMC) igual a $21,5 \mathrm{~kg} / \mathrm{m}^{2}$. Exames laboratoriais iniciais estão descritos na Tabela 1. Distúrbios hidroeletrolíticos foram corrigidos por via endovenosa.

Tabela 1: Resultados de exames laboratoriais na admissão à UTI.

\begin{tabular}{lcc}
\hline \multicolumn{1}{c}{ Exame laboratorial } & Resultado & Valor de referência \\
\hline Hemoglobina & $8,3 \mathrm{~g} / \mathrm{dL}$ & 11,6 a $15,6 \mathrm{~g} / \mathrm{dL}$ \\
Leucócitos & $7870 / \mathrm{mcgL}$ & 3600 a $11000 / \mathrm{mcgL}$ \\
Índice internacional normalizado para protrombina & 0,91 & Até 1,2 \\
Albumina & $2,1 \mathrm{~g} / \mathrm{dL}$ & 3,5 a $5,2 \mathrm{~g} / \mathrm{dL}$ \\
Creatinina & $0,38 \mathrm{mg} / \mathrm{dL}$ & 0,51 a $1,2 \mathrm{mg} / \mathrm{dL}$ \\
Potássio & $3,2 \mathrm{mEq} / \mathrm{L}$ & 3,5 a $5,5 \mathrm{mEq} / \mathrm{L}$ \\
Magnésio & $1,3 \mathrm{mg} / \mathrm{dL}$ & 1,6 a $2,6 \mathrm{mg} / \mathrm{dL}$ \\
Fósforo & $1,2 \mathrm{mg} / \mathrm{dL}$ & 2,5 a $4,5 \mathrm{mg} / \mathrm{dL}$ \\
Creatinina fosfoquinase & $496 \mathrm{U} / \mathrm{L}$ & Até $170 \mathrm{U} / \mathrm{L}$ \\
Glicose & $135 \mathrm{mg} / \mathrm{dL}$ & 70 a $100 \mathrm{mg} / \mathrm{dL}$ \\
Cálcio (corrigido para albumina) & $9,1 \mathrm{mg} / \mathrm{dL}$ & 8,6 a $10 \mathrm{mg} / \mathrm{dL}$ \\
Sódio & $145 \mathrm{mmol} / \mathrm{L}$ & 135 a $145 \mathrm{mmol} / \mathrm{L}$ \\
Bilirrubina total & $0,3 \mathrm{mg} / \mathrm{dL}$ & 0,2 a $1 \mathrm{mg} / \mathrm{dL}$ \\
Aspartato aminotransferase & $82 \mathrm{U} / \mathrm{L}$ & 0 a $32 \mathrm{U} / \mathrm{L}$ \\
Alanina aminotransferase & $75 \mathrm{U} / \mathrm{L}$ & 0 a $33 \mathrm{U} / \mathrm{L}$ \\
Vitamina B12 & $540 \mathrm{pg} / \mathrm{mL}$ & Acima de $211 \mathrm{pg} / \mathrm{mL}$ \\
Anticorpos contra vírus da imunodeficiência humana & Não reagente & Não reagente \\
\hline
\end{tabular}

A paciente foi então submetida à tomografia computadorizada de crânio (TCC), a qual não evidenciou particularidades. Procedeu-se à coleta liquórica mediante punção lombar (PL): presença de 1 leucócito, glicose de $80 \mathrm{mg} / \mathrm{dL}$ (glicemia= $135 \mathrm{mg} / \mathrm{dL}$ ), proteinorraquia de $15 \mathrm{mg} / \mathrm{dL}$ [limite superior $=50 \mathrm{mg} / \mathrm{dL}$ ]) e ausência de crescimento de fungos ou bactérias. Pesquisa de vírus em líquor não foi realizada.

Devido hipótese de catatonia maligna, foi transferida a outra instituição de alta complexidade com o intuito de realização de eletroconvulsoterapia. Foi realizado eletroencefalograma, que descartou 
paroxismos epileptiformes e observou traçado pouco reativo à estimulação, compatível com encefalopatia difusa moderada. Procedeu-se à realização de ressonância magnética (RM) de crânio (Figuras 1 e 2), que evidenciou áreas de hipersinal T2/FLAIR acometendo as porções mediais dos tálamos, mesencéfalo, corpos mamilares, placa quadrigeminal, região periaquedutal, corpo estriado e uncus, algumas com restrição à difusão da água. As regiões adjacentes ao terceiro ventrículo e aqueduto apresentaram importante realce pelo gadolíneo. Os achados imagéticos foram compatíveis com distúrbio de natureza tóxico-metabólica, sendo EW a principal hipótese.

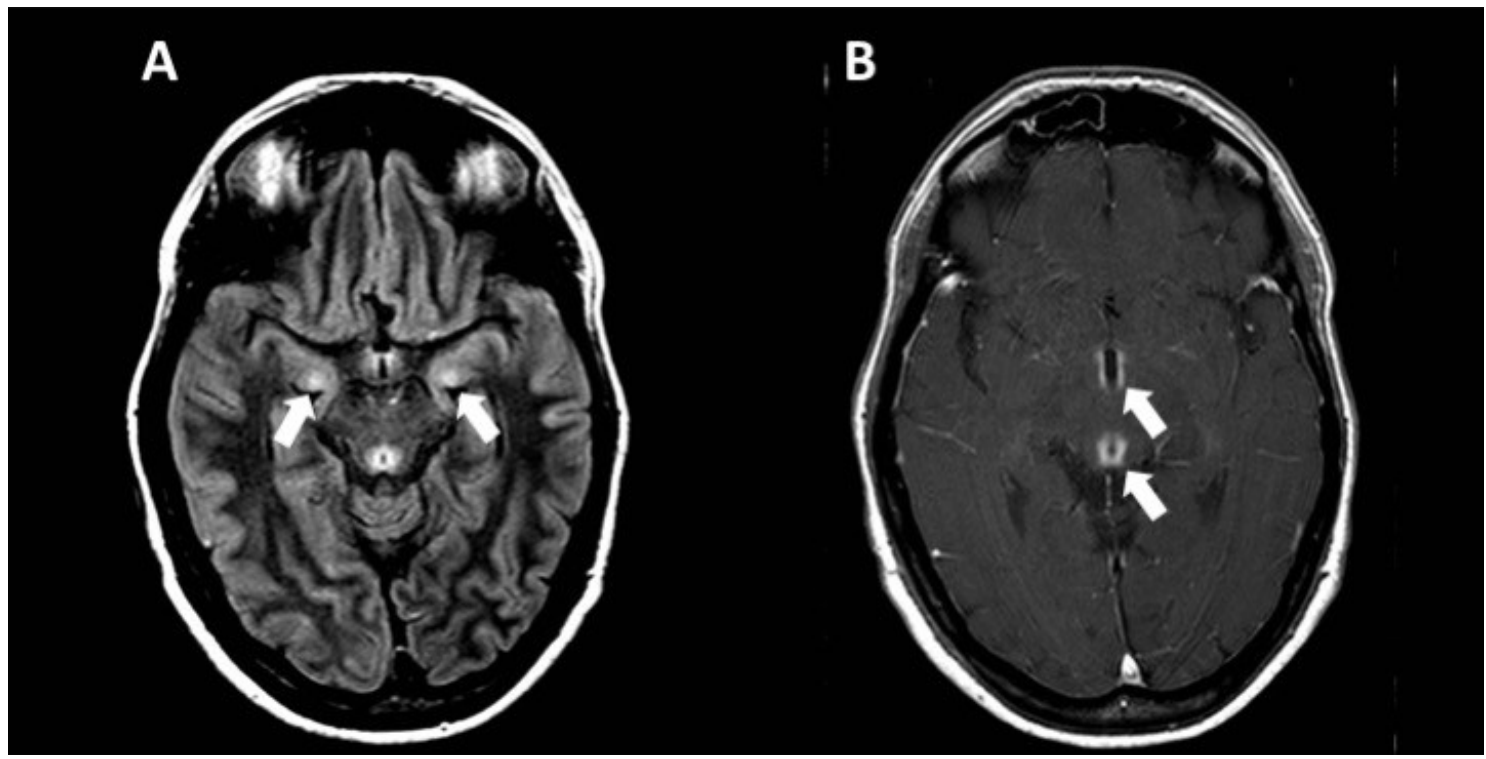

Figura 1: A: Aumento da intensidade de sinal em tálamo dorsomedial, corpos mamilares [seta] e substância cinzenta periaquedutal em T2/Flair; B: Marcado realce pelo contraste em T1 [seta].

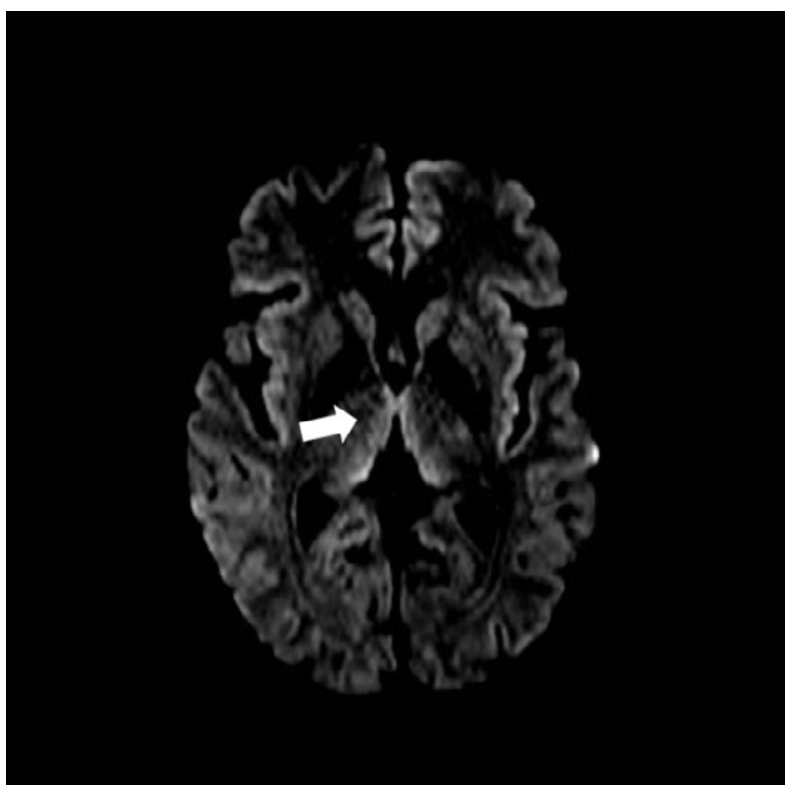

Figura 2: Tálamo dorsomedial e substância cinzenta periaquedutal [seta] apresentando restrição à difusão.

Foi iniciada reposição agressiva de tiamina por via endovenosa, inicialmente na dose de
$500 \mathrm{mg}$ de 8 em 8 horas por 48 horas, seguida de redução para $250 \mathrm{mg}$ diariamente. Como os distúrbios hidroeletrolíticos já haviam sido corrigidos, optou-se pela manutenção do aporte nutricional pleno. Entretanto, o coma profundo irreversível persistiu. A paciente evoluiu para óbito por choque séptico pulmonar e disfunção de múltiplos órgãos no $30^{\circ}$ dia de internação.

\section{DISCUSSÃO}

EW é uma condição neurológica rara, aguda ou subaguda, frequentemente devastadora e de diagnóstico tardioº ${ }^{2}$. Caracterizada pela deficiência de tiamina (vitamina B1), que é uma vitamina hidrossolúvel e cofator essencial para enzimas envolvidas na glicólise e ciclo de Krebs, existe crescente atenção para pacientes criticamente enfermos ${ }^{5}$, muito embora ainda reconhecidamente mais prevalente em alcoolistas - risco de $30 \%$ versus $6 \%$ em não alcoolistas ${ }^{2}$. O diagnóstico da síndrome é eminentemente clínico e requer a presença de dois de quadro sinais: i) déficit nutricional, ii) sinais oculares, iii) disfunção cerebelar, e iv) alteração do estado mental ou memória. A tríade clássica 
é mais comumente exibida por alcoolistas ${ }^{2}$. Dosagem direta de tiamina, atualmente, é possível e viável ${ }^{6}$, porém não amplamente disponível e podendo ser falsamente em casos de mutações genéticas individuais ${ }^{7}$. RM de crânio é o exame de eleição para o diagnóstico, tipicamente exibindo lesões simétricas em tálamo, corpos mamilares, placa tectônica e área periaquedutal. Pacientes não alcoolistas frequentemente apresentam envolvimento atípico de cerebelo, núcleos da base e córtex cerebral ${ }^{2,8}$. Acometimento cortical indica prognóstico neurológico reservado 8 .

SR consiste no desarranjo metabólico observado em pacientes desnutridos ou com baixa ingesta alimentar recente que são submetidos ao reinício de nutrição enteral ou parenteral. A oferta abrupta de nutrientes a pacientes sob risco ocasiona uma alteração rápida de eletrólitos no ambiente extracelular, que agora adentram na célula para serem utilizados nas rotas metabólicas e, em casos extremos, podem culminar em arritmias cardíacas fatais ${ }^{5}$. O desencadeamento da SR promove o desenvolvimento de deficiência de tiamina justamente pela aceleração dos processos enzimáticos envolvidos na respiração celular, e invariavelmente os pacientes também sob risco de carência de vitamina B1 são os mesmos sob risco de $\mathrm{SR}^{4}$. Embora o diagnóstico de $\mathrm{SR}$ ainda seja fonte de controvérsia, a hipofosfatemia, quando aliada às condições clínicas expostas na Tabela 2, é um marcador razoavelmente confiável de sua presença ${ }^{5}$.

Tabela 2: Critérios propostos pelo consenso da American Society for Parenteral and Enteral Nutrition (ASPEN) para identificação de pacientes em risco de síndrome de realimentação ${ }^{5}$.

\begin{tabular}{ll}
\hline Risco moderado - 2 critérios abaixo são necessários: & Risco significativo - $\mathbf{1}$ critério abaixo é necessário: \\
\hline IMC menor que $18,5 \mathrm{~kg} / \mathrm{m}^{2}$ & IMC menor que $16 \mathrm{~kg} / \mathrm{m}^{2}$ \\
$\begin{array}{l}\text { Perda não intencional de peso maior que 5\% no último } \\
\text { mês }\end{array}$ & $\begin{array}{l}\text { Perda não intencional de peso maior que } 7,5 \% \text { nos } \\
\text { úlimos } 3 \text { meses OU maior que } 10 \% \text { nos últimos } 6 \text { meses }\end{array}$ \\
$\begin{array}{l}\text { Mínima ou nenhuma ingestão calórica por 5-6 dias, OU } \\
\text { aporte inferior a } 75 \% \text { do necessário por mais de }\end{array}$ & $\begin{array}{l}\text { Mínima ou nenhuma ingestão calórica por mais de } \\
7 \text { dias, OU aporte inferior a } 50 \% \text { do necessário por }\end{array}$ \\
$\begin{array}{l}7 \text { dias em contexto de doença crítica OU ingesta } \\
\text { calórica inferior a } 75 \% \text { do necessário por mais de }\end{array}$ & $\begin{array}{l}\text { mais de } 5 \text { dias em contexto de doença crítica OU O } \\
\text { ingesta calórica inferior a } 50 \% \text { do necessário por mais } \\
\text { de } 30 \text { dias }\end{array}$
\end{tabular}

Níveis de potássio, magnésio ou fósforo no limite inferior ou discretamente reduzidos previamente ao início da terapia nutricional

Evidência de moderada perda de gordura em subcutâneo e massa muscular

Níveis de potássio, magnésio ou fósforo muito baixos previamente ao início da terapia nutricional

Evidência de grande perda de gordura em subcutâneo e massa muscular

Presença de doença do grupo de risco (vide texto) com descompensação moderada

Presença de doença do grupo de risco (vide texto) com descompensação grave

Estima-se que 2 a 3 semanas de carência de vitamina B1 seja o suficiente para provocar lesões cerebrais ${ }^{3}$. Grupos de risco identificados em indivíduos não etilistas incluem presença de neoplasias malignas, cirurgia bariátrica prévia, hiperêmese gravídica, síndromes intestinais disabsortivas, síndrome da imunodeficiência humana adquirida (SIDA), anorexia nervosa e virtualmente qualquer distúrbio mental que ocasione "greve de fome", tais como Doença de Alzheimer, transtorno do humor bipolar e esquizofrenia ${ }^{2,3,9,10}$.

Tanto a SR quanto a EW devem ser prioritariamente prevenidas com reposição de tiamina imediatamente antes de qualquer infusão de aporte energético, nutricional ou não ${ }^{1-5}$, para tanto requerendo alto grau de suspeição diagnóstica. Além disso, a vigilância de níveis de potássio, magnésio e fósforo são mandatórias até duas vezes ao dia nas fases iniciais de realimentação. A provisão inicial de calorias varia de 5 a $20 \mathrm{kcal} / \mathrm{kg}$ de peso atual e com incrementos parcimoniosos a cada 3-7 dias, de acordo com o comportamento dos níveis de eletrólitos ${ }^{5}$.

\section{Conflitos de Interesse}

Os autores declaram ausência de conflito de interesse na divulgação do presente caso clínico. 


\section{REFERÊNCIAS}

1. Hutcheon DA. Malnutrition-induced Wernicke's encephalopathy following a water-only fasting diet. Nutr Clin Pract. 2015;30(1):92-9.

2. Galvin R, Brathen $G$, Ivashynka A, Hillbom M, Tanasescu R, Leone MA. EFNS guidelines for diagnosis, therapy and prevention of Wernicke encephalopathy. Eur J Neurol. 2010;17(12):1408-18.

3. Sechi G, Serra A. Wernicke's encephalopathy: new clinical settings and recent advances in diagnosis and management. Lancet Neurol. 2007;6(5):442-55.

4. Polegato BF, Pereira AG, Azevedo PS, Costa NA, Zornoff LAM, Paiva SAR, et al. Role of thiamin in health and disease. Nutr Clin Pract. 2019;34(4):558-64.
5. Silva JSV, Seres DS, Sabino K, Adams SC, Berdahl GJ, Citty SW, et al. ASPEN Consensus recommendations for refeeding syndrome. Nutr Clin Pract. 2020;35(2):178-95.

6. Lu J, Frank EL. Rapid HPLC measurement of thiamine and its phosphate esters in whole blood. Clin Chem. 2008;54(5):901-6.

7. Kono S, Miyajima $\mathrm{H}$, Yoshida $\mathrm{K}$, Togawa A, Shirakawa K, Suzuki $H$. Mutations in a thiaminetransporter gene and Wernicke'slike encephalopathy. $N$ Engl J Med. 2009;360(17):1792-4.
8. Manzo G, De Gennaro A, Cozzolino A, Serino A, Fenza G, Manto A. MR imaging findings in alcoholic and nonalcoholic acute Wernicke's encephalopathy: a review. Biomed Res Int. 2014;2014:1-12.

9. Hershkowitz E, Reshef A, Munich $O$, Yosefi B, Markel A. Thiamine deficiency in self-induced refeeding syndrome, an undetected and potentially lethal condition. Case Rep Med. 2014;2014:1-6.

10. Oudman E, Wijnia JW, Oey MJ, van Dam MJ, Postma A. Preventing Wernicke's encephalopathy in anorexia nervosa: a systematic review. Psychiatry Clin Neurosci. 2018;72(10):774-9.

Recebido: 27 maio, 2020 Aceito: 28 ago, 2020 